\title{
EFFECTS OF SOFT GLUON EMISSION ON THE OPPOSITE-SIDE ACOLLINEARITY DISTRIBUTIONS IN $\mathrm{e}^{+} \mathrm{e}^{-}$ANNIHILATION
}

\author{
R. BAIER and K. FEY \\ Department of Theoretical Physics, University of Bielefeld, Germany \\ Received 16 June 1980 \\ (Revised 24 October 1980)
}

\begin{abstract}
The acollinearity of hadrons belonging to back-to-back jets in $\mathrm{e}^{+} \mathrm{e}^{-}$annihilation is investigated in the framework of QCD. The approach is based on the evolution equations for fragmentation functions, and it takes into account the non-perturbative intrinsic transverse momentum. The results are compared with recent data, and satisfactory agreement is obtained.
\end{abstract}

\section{Introduction}

The PLUTO collaboration [1] has recently measured the two-particle acollinearity distributions of charged hadrons in $\mathrm{e}^{+} \mathrm{e}^{-}$annihilation at different energies $Q=7.7-31.6 \mathrm{GeV}$. In this paper we investigate the effects of soft gluon emission on these distributions, when the hadrons belong to opposite jets. Our approach is based on the evolution equations for the fragmentation functions $D_{\mathrm{a}}^{\mathrm{b}}\left(x, \boldsymbol{k}_{\mathrm{T}}, Q^{2}\right)$ for parton a into parton $b$ (with energy fraction $x$ and transverse momentum $\boldsymbol{k}_{\mathrm{T}}$ relative to a). They have been derived by Bassetto et al. [2] in the leading logarithmic approximation (LLA) of QCD. By solving these equations one obtains the $\boldsymbol{k}_{\mathrm{T}}$ dependence by summing the emission of partons in the soft limit to all orders of perturbation theory. This resummed QCD perturbation theory has been applied to transverse momentum spectra first by Parisi and Petronzio [3]. We include the fragmentation of partons into hadrons by assuming that at the end of the hard QCD evolution process each parton transforms independently into hadrons $[4,5]$. Therefore, within the framework of perturbative QCD the parton into hadron fragmentation functions $D_{\mathrm{a}}^{\mathrm{h}}\left(x, \boldsymbol{k}_{\mathrm{T}}, Q^{2}\right)$ for finite $Q^{2}$ can only be calculated in terms of those at some value of $Q_{0}^{2}$, where one either has to use data directiy, or some parametrization in agreement with data. This procedure takes into account the intrinsic non-perturbative transverse momentum of hadrons fragmenting from partons.

The results of our calculations are compared with the data of ref. [1]. We also perform a comparison with the predictions of (i) the parton model (with $Q^{2}$ 
independent fragmentation functions), (ii) the leading double logarithmic approximation, first discussed by Dokshitser et al. [4, 6], and (iii) the lowest-order perturbative calculation [7].

\section{The acollinearity distribution and the evolution equations}

When two hadrons belonging to back-to-back jets are observed inclusively in $\mathrm{e}^{+} \mathrm{e}^{-}$annihilation, they originate from a quark $\mathrm{q}$ and an antiquark $\overline{\mathrm{q}}$ which are initially produced from a virtual photon of mass $Q$. The probability distribution for a quark which decays into a hadron $\mathrm{h}$ with energy fraction $x$ and transverse momentum $\boldsymbol{k}_{\mathrm{T}}$ relative to that quark is denoted by $D_{\mathrm{q}}^{\mathrm{h}}\left(x, \boldsymbol{k}_{\mathrm{T}}, Q^{2}\right)$. Using planar gauges in QCD one can apply (in LLA) the same probabilistic interpretation as in the parton model [4]. Consequently the cross section for the considered hadron production is expressed by a product of $D$-functions,

$$
\begin{aligned}
\frac{1}{\sigma} \frac{\mathrm{d} \boldsymbol{\sigma}^{\mathrm{h}_{\mathrm{a}} \mathrm{h}_{\mathrm{b}}}}{\mathrm{d} x_{\mathrm{a}} \mathrm{d} x_{\mathrm{b}} \mathrm{d}^{2} \boldsymbol{p}_{\mathrm{T}}}= & \frac{1}{\sum_{\mathrm{q}} e_{\mathrm{q}}^{2}} \int \mathrm{d}^{2} \boldsymbol{k}_{\mathrm{Ta}} \mathrm{d}^{2} \boldsymbol{k}_{\mathrm{Tb}} \delta\left(\boldsymbol{p}_{\mathrm{T}}-\boldsymbol{k}_{\mathrm{Ta}} / x_{\mathrm{a}}-\boldsymbol{k}_{\mathrm{Tb}} / x_{\mathrm{b}}\right) \\
& \times\left\{\sum_{\mathrm{q}} e_{\mathrm{q}}^{2} D_{\mathrm{q}}^{\mathrm{h}_{\mathrm{a}}}\left(x_{\mathrm{a}}, \boldsymbol{k}_{\mathrm{Ta}}, Q^{2}\right) D_{\overline{\mathrm{q}}}^{\mathrm{h}_{\mathrm{h}}}\left(x_{\mathrm{b}}, \boldsymbol{k}_{\mathrm{Tb}}, Q^{2}\right)+(\mathrm{q} \leftrightarrow \overline{\mathrm{q}})\right\},
\end{aligned}
$$

where $e_{\mathrm{q}}$ is the quark charge and $\boldsymbol{p}_{\mathrm{T}}$ is the (scaled) relative transverse momentum of the hadrons $h_{a}$ and $h_{b}$.

In the experiment of ref. [1] the energy-weighted distribution as a function of the acollinearity angle $\theta$ is measured. We therefore define the following distribution, which is normalized to one:

$$
\frac{\mathrm{d} w}{\mathrm{~d} \sin ^{2} \frac{1}{2} \theta} \equiv \frac{1}{2} \sum_{\mathrm{h}_{\mathrm{a}}, \mathrm{h}_{\mathrm{b}}} \int_{0}^{1} x_{\mathrm{a}} \mathrm{d} x_{\mathrm{a}} x_{\mathrm{b}} \mathrm{d} x_{\mathrm{b}} \frac{1}{\sigma} \frac{\mathrm{d} \boldsymbol{\sigma}^{\mathrm{h}_{\mathrm{a}} \mathrm{h}_{\mathrm{b}}}}{\mathrm{d} x_{\mathrm{a}} \mathrm{d} x_{\mathrm{b}} \mathrm{d} \sin ^{2} \frac{1}{2} \theta}
$$

In the following we only consider small angles $\theta$ for which the relation $p_{\mathrm{T}}^{2} \simeq Q^{2} \sin ^{2} \frac{1}{2} \theta$ holds.

In terms of the moments of the distributions $D_{\mathrm{q}}^{\mathrm{h}}$ defined by the Fourier-Mellin transform [2]

$$
d_{\mathrm{q}}^{\mathrm{h}}\left(n, b, Q^{2}\right)=\int_{0}^{1} \mathrm{~d} x x^{n-1} \int \mathrm{d}^{2} \boldsymbol{k}_{\mathrm{T}} \exp \left(-i \boldsymbol{k}_{\mathrm{T}} \cdot \boldsymbol{b} / x\right) D_{\mathrm{q}}^{\mathrm{h}}\left(x, \boldsymbol{k}_{\mathrm{T}}, Q^{2}\right),
$$


the cross-section equation (2), together with eq. (1), becomes

$$
\begin{aligned}
\frac{\mathrm{d} w}{\mathrm{~d} \sin ^{2} \frac{1}{2} \theta}= & \frac{1}{4} \frac{1}{\sum_{\mathrm{q}} e_{\mathrm{q}}^{2}} \int_{0}^{\infty} \mathrm{d}(Q b) Q b J_{0}\left(Q b \sin \frac{1}{2} \theta\right) \\
& \times\left\{\sum_{\mathrm{q}} e_{\mathrm{q}}^{2} \sum_{\mathrm{h}_{\mathrm{a}}} d_{\mathrm{q}}^{\mathrm{h}_{\mathrm{a}}}\left(n=2, b, Q^{2}\right) \sum_{\mathrm{h}_{\mathrm{b}}} d_{\overline{\mathrm{q}}}^{\mathrm{h}_{\mathrm{b}}}\left(n=2, b, Q^{2}\right)+(\mathrm{q} \leftrightarrow \overline{\mathrm{q}})\right\},
\end{aligned}
$$

where $J_{0}$ is the Bessel function of the first kind. In order to evaluate this expression one has to know the $Q^{2}$ dependence of the moments $d_{\mathrm{q}}^{\mathrm{h}}$ for all impact parameters $b$. For this purpose we first use the evolution equations for quark and gluons, as they are derived in ref. [2] in LLA by summing dressed ladder diagrams [4]. The equations read for $n>1$,

$$
\frac{\mathrm{d}}{\mathrm{d} t} d_{\mathrm{a}}^{\mathrm{b}}\left(n, b, Q^{2}\right)=\frac{\alpha_{\mathrm{s}}(t)}{2 \pi} \sum_{\mathrm{c}} \int_{0}^{1} \mathrm{~d} z z^{n-1} P_{\mathrm{a}}^{\mathrm{c}}(z) d_{\mathrm{c}}^{\mathrm{b}}\left(n, b / z, Q^{2}\right) J_{0}\left(Q b \sqrt{\frac{1-z}{z}}\right),
$$

with $t=\ln \left(Q^{2} / \Lambda^{2}\right), \alpha_{\mathrm{s}}(t)=4 \pi /\left(\beta_{0} t\right), \beta_{0}=11-\frac{2}{3} n_{\mathrm{f}}$ for $n_{\mathrm{f}}$ flavors, and $(\mathrm{a}, \mathrm{b}, \mathrm{c})=\mathrm{q}$ or gluon g. The functions $P_{\mathrm{a}}^{\mathrm{c}}$ are the Altarelli-Parisi densities [8]. The reason for the formulation in the impact parameter space is the following: the transverse momentum $\boldsymbol{k}_{\mathrm{T}}$ of the final parton $\mathrm{b}$ is the sum of the transverse momenta of the partons which are emitted during the jet evolution. The emitted partons are not forced to have transverse momenta less than $\left|\boldsymbol{k}_{\mathrm{T}}\right|$, as it is the case in the leading double logarithmic approximation [3, 4].

As a next step we incorporate phenomenologically the hadronization of quarks and gluons. We assume that the fragmentation functions $D_{\mathrm{a}}^{\mathrm{h}}\left(x, \boldsymbol{k}_{\mathrm{T}}, Q^{2}\right)$ for a $=\mathrm{q}$ or $\mathrm{g}$ into $\mathrm{h}$ are obtained from the distributions $D_{\mathrm{a}}^{\mathrm{b}}$ by the following convolution:

$$
D_{\mathrm{a}}^{\mathrm{h}}\left(x, \boldsymbol{k}_{\mathbf{T}}, Q^{2}\right)=\sum_{\mathrm{b}} \int_{x}^{1} \frac{\mathrm{d} y}{y} \int \mathrm{d}^{2} \boldsymbol{q}_{\mathrm{T}} D_{\mathrm{a}}^{\mathrm{b}}\left(x / y, \boldsymbol{q}_{\mathrm{T}}, Q^{2}\right) \tilde{D}_{\mathrm{b}}^{\mathrm{h}}\left(y, \boldsymbol{k}_{\mathrm{T}}-y \boldsymbol{q}_{\mathrm{T}}\right) .
$$

The theoretically unknown functions $\tilde{D}_{\mathrm{b}}^{\mathrm{h}}$ are independent of $Q^{2}$ and are supposed to describe the limited transverse momentum of $h$ with respect to $b$. They are assumed to satisfy the following relation due to the requirement of energy conservation:

$$
\sum_{\mathrm{h}} \int_{0}^{1} \mathrm{~d} x \int \mathrm{d}^{2} \boldsymbol{k}_{\mathrm{T}} x \tilde{D}_{\mathrm{b}}^{\mathrm{h}}\left(x, \boldsymbol{k}_{\mathrm{T}}\right)=1
$$


As already mentioned before the ansatz, eq. (6) assumes an independent hadronization of the parton $b$. It is generally applied for the $\boldsymbol{k}_{\mathbf{T}}$ integrated fragmentation functions [4]. As a consequence of eq. (6) it follows that the moments $d_{\mathrm{a}}^{\mathrm{h}}\left(n, b, Q^{2}\right)$ obey the same evolution equations as the moments $d_{\mathrm{a}}^{\mathrm{b}}$, except that in eq. (5) the constituent $b$ is replaced by the hadron $h$.

In order to proceed we define in the standard way [9] the non-singlet moments

$$
d_{\mathrm{NS}}\left(n, b, Q^{2}\right)=d_{\mathrm{q}_{i}}^{\mathrm{h}}\left(n, b, Q^{2}\right)-d_{\mathrm{q}}^{\mathrm{h}}\left(n, b, Q^{2}\right),
$$

$q_{i} \neq q_{j}$, and the singlet moments

$$
d_{\mathrm{S}}^{\mathrm{h}}\left(n, b, Q^{2}\right)=\sum_{i=1}^{2 n_{\mathrm{i}}} d_{\mathrm{q}_{i}}^{\mathrm{h}}\left(n, b, Q^{2}\right)
$$

The case $b=0$, i.e., the moments of the $\boldsymbol{k}_{\mathrm{T}}$ integrated fragmentation functions, is discussed in ref. [10]. The $Q^{2}$ evolution of $d_{\mathrm{NS}}^{\mathrm{h}}$ is then given by

$$
\frac{\mathrm{d}}{\mathrm{d} t} d_{\mathrm{NS}}^{\mathrm{h}}\left(n, b, Q^{2}\right)=\frac{\alpha_{\mathrm{s}}(t)}{2 \pi} \int_{0}^{1} \mathrm{~d} z z^{n-1} P_{\mathrm{qq}}(z) d_{\mathrm{NS}}^{\mathrm{h}}\left(n, b / z, Q^{2}\right) J_{0}\left(Q b \sqrt{\frac{1-z}{z}}\right),
$$

where

$$
P_{\mathrm{qq}}=c_{\mathrm{F}}\left(\frac{1+z^{2}}{1-z}\right)_{+}, \quad c_{\mathrm{F}}=\frac{4}{3}
$$

As proposed in ref. [2], this equation can be solved approximately by first splitting the r.h.s. into two parts,

$$
\begin{aligned}
\int_{0}^{1} \mathrm{~d} z z^{n-1} P_{\mathrm{qq}}(z) d_{\mathrm{NS}}^{\mathrm{h}}\left(n, b, Q^{2}\right)+\int_{0}^{1} \mathrm{~d} z z^{n-1} P_{\mathrm{qq}}(z) \\
\\
\quad\left[d_{\mathrm{NS}}^{\mathrm{h}}\left(n, b / z, Q^{2}\right) J_{0}\left(Q b \sqrt{\frac{1-z}{z}}\right)-d_{\mathrm{NS}}^{\mathrm{h}}\left(n, b, Q^{2}\right)\right],
\end{aligned}
$$

and second by putting $z=1$ in all slowly varying expressions of the second term. Then the r.h.s. of eq. (8) reduces to

$$
\frac{\alpha_{\mathrm{s}}(t)}{2 \pi} d_{\mathrm{NS}}^{\mathrm{h}}\left(n, b, Q^{2}\right)\left[\int_{0}^{1} \mathrm{~d} z z^{n-1} P_{\mathrm{qq}}(z)+\int_{0}^{1} \mathrm{~d} z \frac{2 c_{\mathrm{F}}}{\left(1-z+\varepsilon^{\prime}\right)}\left(J_{0}(Q b \sqrt{ } 1-\bar{z})-1\right)\right]
$$


The parameter $\varepsilon^{\prime}$ in the singular function $\left(1-z+\varepsilon^{\prime}\right)^{-1}$ is introduced in order to kccp track of the planar gauge chosen in our problem. With this approximation only the soft gluon emission is taken into account in the second term; it is independent in impact parameter space, as it is the case for the soft photon emission in QED [3]. The first term in eq. (9) is responsible for the scale-breaking behaviour of the $\boldsymbol{k}_{\mathrm{T}}$ integrated fragmentation functions. From eqs. (8), (9) one obtains the solution

$$
d_{\mathrm{NS}}^{\mathrm{h}}\left(n, b, Q^{2}\right)=d_{\mathrm{NS}}^{\mathrm{h}}\left(n, b, Q_{0}^{2}\right) \exp \left[A_{n}^{\mathrm{NS}} s\right] F_{\mathrm{qq}}\left(Q^{2}, Q_{0}^{2}, b\right),
$$

where

$$
s=\frac{2}{\beta_{0}} \ln \alpha_{\mathrm{s}}\left(Q_{0}^{2}\right) / \alpha_{\mathrm{s}}\left(Q^{2}\right), \quad A_{n}^{\mathrm{NS}}=\int_{0}^{1} \mathrm{~d} z z^{n-1} P_{\mathrm{qq}}(z)
$$

The non-singlet form factor $F_{\mathrm{qq}}[2]$ is given by

$$
F_{\mathrm{qq}}\left(Q^{2}, Q_{0}^{2}, b\right)=\exp \left[-\frac{c_{\mathrm{F}}}{2 \pi} \int_{Q_{0}^{2}}^{Q^{2}} \mathrm{~d} k^{2} \frac{\alpha_{\mathrm{s}}\left(k^{2}\right)}{k^{2}} f_{e}(b k)\right]
$$

with

$$
f_{\varepsilon}(b k)=4 \int_{0}^{b k} \frac{\mathrm{d} q}{(q+\varepsilon)}\left(1-J_{0}(q)\right), \quad \varepsilon=\varepsilon^{\prime} b^{2} k^{2} / q
$$

The coupled moment equations for $d_{\mathrm{s}}^{\mathrm{h}}$ and $d_{\mathrm{g}}^{\mathrm{h}}$ read, when the same approximations as above [eq. (9)] are performed,

$$
\frac{\mathrm{d}}{\mathrm{d} t}\left(\begin{array}{l}
d_{\mathrm{S}}^{\mathrm{h}}\left(n, b, Q^{2}\right) \\
d_{\mathrm{g}}^{\mathrm{h}}\left(n, b, Q^{2}\right)
\end{array}\right)=\frac{\alpha_{\mathrm{s}}(t)}{2 \pi}\left(\begin{array}{cc}
A_{n}^{\mathrm{NS}}-c_{\mathrm{F}} f_{\varepsilon}(b Q) & 2 n_{\mathrm{f}} A_{n}^{\mathrm{gq}} \\
A_{n}^{\mathrm{qg}} & A_{n}^{\mathrm{gg}}-c_{\mathrm{A}} f_{\varepsilon}(b Q)
\end{array}\right)\left(\begin{array}{l}
d_{\mathrm{S}}^{\mathrm{h}}\left(n, b, Q^{2}\right) \\
d_{\mathrm{g}}^{\mathrm{h}}\left(n, b, Q^{2}\right)
\end{array}\right)
$$

with the standard notation for the anomalous dimensions $A_{n}$, and $c_{\mathrm{A}}=3[9,10]$. A convenient method to diagonalize this system is to use the Pauli matrices $\sigma$ and to rewrite eq. (12) symbolically as

$$
\frac{\mathrm{d}}{\mathrm{d} t}(d(t))=\left(A_{0}(t) \mathrm{\Omega}+\boldsymbol{A}(t) \cdot \boldsymbol{\sigma}\right)(d(t)),
$$

which has the solution

$$
(d(t))=\exp \left[\int_{t_{0}}^{t}\left(A_{0}\left(t^{\prime}\right) \mathbb{1}+A\left(t^{\prime}\right) \cdot \boldsymbol{\sigma}\right) \mathrm{d} t^{\prime}\right]\left(d\left(t_{0}\right)\right)
$$


By expanding the exponential and using the properties of the Pauli matrices the explicit solution of eq. (12) is obtained:

$$
\begin{aligned}
& {\left[\begin{array}{l}
d_{\mathrm{S}}^{\mathrm{h}}\left(n, b, Q^{2}\right) \\
d_{\mathrm{g}}^{\mathrm{h}}\left(n, b, Q^{2}\right)
\end{array}\right)} \\
& \quad=\left[\begin{array}{cc}
\left(1-\alpha_{n}\right) \mathrm{e}^{A_{n}^{+} s}+\alpha_{n} \mathrm{e}^{A_{n}^{-} s} & \beta_{n}\left(\mathrm{e}^{A_{n}^{-} s}-\mathrm{e}^{A_{n}^{+} s}\right) \\
\gamma_{n}\left(\mathrm{e}^{A_{n}^{-} s}-\mathrm{e}^{A_{n}^{+} s}\right) & \alpha_{n} \mathrm{e}^{A_{n}^{+} s}+\left(1-\alpha_{n}\right) \mathrm{e}^{A_{n} s}
\end{array}\right)\left(\begin{array}{l}
d_{\mathrm{S}}^{\mathrm{h}}\left(n, b, Q_{0}^{2}\right) \\
d_{\mathrm{g}}\left(n, b, Q_{0}^{2}\right)
\end{array}\right],
\end{aligned}
$$

where

$$
\begin{aligned}
& A_{n}^{ \pm}=\frac{1}{2}\left(\gamma_{n}^{\mathrm{q}}+\gamma_{n}^{\mathrm{g}} \mp\left[\left(\gamma_{n}^{\mathrm{q}}-\gamma_{n}^{\mathrm{g}}\right)^{2}+8 n_{\mathrm{f}} A_{n}^{\mathrm{qg}} A_{n}^{\mathrm{gq}}\right]^{1 / 2}\right), \\
& \gamma_{n}^{\mathrm{q}}=A_{n}^{\mathrm{NS}}-\frac{c_{\mathrm{F}}}{2 \pi s} \int_{Q_{0}^{2}}^{Q^{2}} \mathrm{~d} k^{2} \frac{\alpha_{\mathrm{s}}\left(k^{2}\right)}{k^{2}} f_{\varepsilon}(b k), \\
& \gamma_{n}^{\mathrm{g}}=A_{n}^{\mathrm{gg}}-\frac{c_{A}}{2 \pi s} \int_{Q_{0}^{2}}^{Q^{2}} \mathrm{~d} k^{2} \frac{\alpha_{\mathrm{s}}\left(k^{2}\right)}{k^{2}} f_{\varepsilon}(b k), \\
& \alpha_{n}=\frac{A_{n}^{+}-\gamma_{n}^{\mathrm{q}}}{A_{n}^{+}-A_{n}^{-}}, \quad \beta_{n}=-\frac{2 n_{\mathrm{f}} A_{n}^{\mathrm{gq}}}{A_{n}^{+}-A_{n}^{-}}, \quad \gamma_{n}=\frac{A_{n}^{\mathrm{qg}}}{2 n_{\mathrm{f}} A_{n}^{\mathrm{gq}}} \beta_{n} .
\end{aligned}
$$

For $b=0$ this solution coincides with the one of ref. [10].

In a recent paper by Amati et al. [11] it is conjectured that the evolution equations given by eq. (5) are to be modified, since the right choice of the argument of the running coupling constant is $\alpha_{s}\left(Q^{2} z(1-z)\right)$ instead of $\alpha_{s}\left(Q^{2}\right)$. By rescaling the argument of $\alpha_{\mathrm{s}}$ large corrections due to phase space constraints are resumed. When one adopts this point of view the eqs. (10)-(15) have to be changed accordingly: the expression [eq. (11)]

$$
\int_{Q_{0}^{2}}^{Q^{2}} \mathrm{~d} k^{2} \frac{\alpha_{\mathrm{s}}\left(k^{2}\right)}{k^{2}} f_{\varepsilon}(b k),
$$

is replaced by

$$
\int_{Q_{0}^{2}}^{Q^{2}} \frac{\mathrm{d} k^{2}}{k^{2}} \int_{b Q_{0}^{\prime}}^{b k} \frac{4 \alpha_{\mathrm{s}}\left(q^{2} / b^{2}\right)}{(q+\varepsilon)}\left(1-J_{0}(q)\right) \mathrm{d} q
$$

where the infrared cut-off $Q_{0}^{\prime}$ has to be introduced, with $\alpha_{s}\left(Q_{0}^{\prime 2}\right) / 2 \pi \lesssim 1$ [11]. The corresponding modifications of the anomalous dimensions $A_{n}$, which appear mainly 
for large $n$, will be neglected, since we are only interested in the moments for $n=2$ [eq. (4)].

After having solved the evolution equations for $d_{\mathrm{q}, \mathrm{g}}^{\mathrm{h}}$ in terms of initial values at some $Q_{0}^{2}$, we are now in the position to evaluate $\mathrm{d} w / \mathrm{d} \sin ^{2} \frac{1}{2} \theta$ given by eq. (4). Because of the implicit gauge dependence of the factorized ansatz in eq. (4) and of the solutions for the moments $d_{\mathrm{q}, \mathrm{g}}^{\mathrm{h}}$ the gauge parameter $\varepsilon$ has to be fixed ${ }^{\star}$. In the light-like gauge one can put $\varepsilon \rightarrow 0$ for the quark line and correspondingly $\varepsilon \rightarrow \infty$ for the antiquark line [12], i.e., the soft gluons are then only emitted from the initial quark. With respect to the input functions $\Sigma_{\mathrm{h}} d_{\mathrm{q}, \mathrm{g}}^{\mathrm{h}}\left(n=2, b, Q_{0}^{2}\right)$ we simplify the following discussion and numerical analysis by assuming that they are independent of the species of the constituents, namely

$$
\sum_{\mathrm{h}} d_{\mathrm{q}}^{\mathrm{h}}\left(n=2, b, Q_{0}^{2}\right)=\sum_{\mathrm{h}} d_{\mathrm{g}}^{\mathrm{h}}\left(n=2, b, Q_{0}^{2}\right) \equiv d\left(b, Q_{0}^{2}\right)
$$

For a parametrization of $d\left(b, Q_{0}^{2}\right)$ we use the following fragmentation function of quarks into hadrons:

$$
\sum_{\mathrm{h}} D_{\mathrm{q}}^{\mathrm{h}}\left(x, \boldsymbol{k}_{\mathrm{T}}\right)=3 \frac{(1-x)^{2}}{x} \frac{2 \exp \left(-2 k_{\mathrm{T}} /\left\langle\boldsymbol{k}_{\mathrm{T}}\right\rangle\right)}{\pi\left\langle\boldsymbol{k}_{\mathrm{T}}\right\rangle^{2}} .
$$

This ansatz should describe fairly well inclusive hadron spectra in $\mathrm{e}^{+} \mathrm{e}^{-}$annihilation in the energy range for which clean two-jets events are observed. Since the three-jet structure starts to emerge around $Q \simeq 10-15 \mathrm{GeV}$ [13], the reference energy $Q_{0}$ should be taken below these energies. For our calculations we choose $Q_{0}=9.4 \mathrm{GeV}$. In the data for $\mathrm{e}^{+} \mathrm{e}^{-} \rightarrow$ hadrons a correlation of the $x$ and $\boldsymbol{k}_{\mathrm{T}}$ dependence (seagull effect) is observed [14]. Because of the energy weighting in the acollinearity distribution [eq. (2)] we therefore expect that the effective $\left\langle\boldsymbol{k}_{\mathrm{T}}\right\rangle$ in eq. (18) has to be larger than the standard value of $0.35 \mathrm{GeV} / c$. We approximate $\left\langle\boldsymbol{k}_{\mathrm{T}}\right\rangle$ by $\left\langle\boldsymbol{k}_{\mathrm{T}}(x)\right\rangle$ at $x \simeq\langle x\rangle \simeq 0.2$, which is the average energy fraction of the data of ref. [1]. From measurements [14] of $\left\langle\boldsymbol{k}_{\mathrm{T}}^{2}(x)\right\rangle$ we estimate $\left\langle\boldsymbol{k}_{\mathrm{T}}(x \sim 0.2)\right\rangle \simeq 0.45-0.50$ $\mathrm{GeV} / c$ at $Q_{0}=9.4 \mathrm{GeV}$. From the transform eq. (3) and from eq. (18) we obtain the input function in the impact parameter space:

$$
\begin{aligned}
d\left(b, Q_{0}^{2}\right) & =d\left(\tilde{b}=b\left\langle\boldsymbol{k}_{\mathrm{T}}\right\rangle\right) \\
& =9 \tilde{b}^{2} \ln \frac{1+\sqrt{1+\tilde{b}^{2}}}{\tilde{b}}-6 \tilde{b}+8 \tilde{b}^{3}+\left(1-8 \tilde{b}^{2}\right) \sqrt{1+\tilde{b}^{2}} .
\end{aligned}
$$

At $\tilde{b}=0$ the function $d$ equals one, and for large $\tilde{b}$ it decreases like $d \sim \tilde{b}^{3}$.

\footnotetext{
* For the result given in eq. (23) below the independence on the gauge parameter can be explicitly checked.
} 


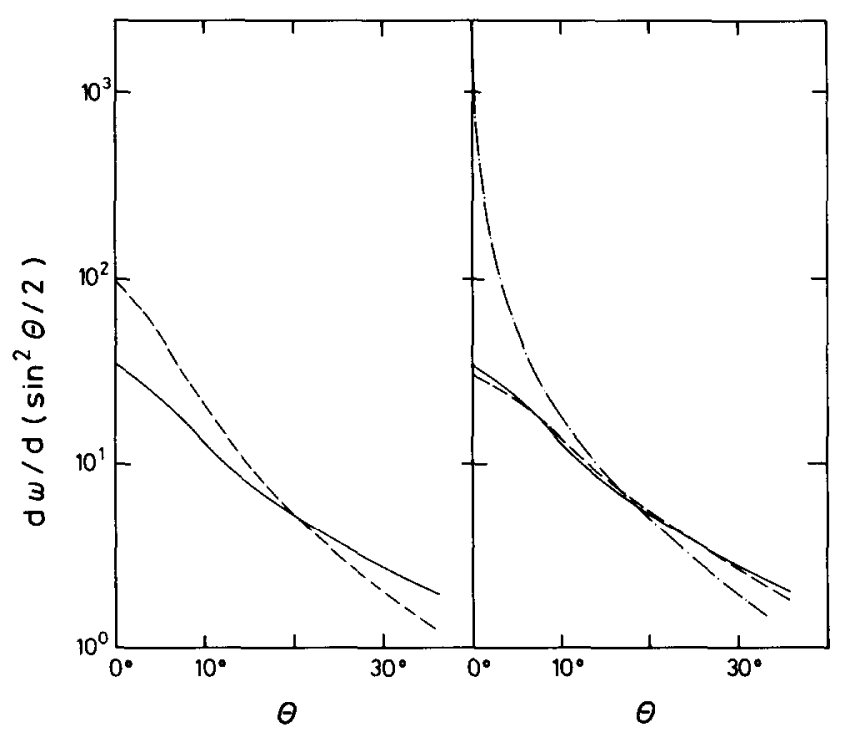

Fig. 1. The dependence of $\mathrm{d} w / \mathrm{d}\left(\sin ^{2} \frac{1}{2} \theta\right)$ on $\theta$ at $Q=30 \mathrm{GeV}$. (a) The solid curve shows the result of our QCD calculation [eq. (20)], the dashed one results from the parton model [eq. (21)]. (b) The solid curve is the same as in (a), the dashed one follows from the modified evolution equations of ref. [11] with eq. (16) and $Q_{0}^{\prime}=1 \mathrm{GeV}$. The dashed-dotted curve shows the QED-like result $\mathrm{d} w^{\mathrm{QED}} / \mathrm{d}\left(\sin ^{2} \frac{1}{2} \theta\right)$ of eq. (23).

The final result of our approach for the energy-weighted acollinearity cross section is given by

$$
\begin{aligned}
\frac{\mathrm{d} w}{\mathrm{~d} \sin _{2}^{2} \theta}= & \frac{1}{2} \int_{0}^{\infty} \mathrm{d}(b Q) b Q J_{0}\left(b Q \sin \frac{1}{2} \theta\right)\left(d\left(b, Q_{0}^{2}\right)\right)^{2} \\
& \times\left[\left(1-\alpha_{2}-\frac{\beta_{2}}{2 n_{\mathrm{f}}}\right) \exp \left(A_{2}^{+} s\right)+\left(\alpha_{2}+\frac{\beta_{2}}{2 n_{\mathrm{f}}}\right) \exp \left(A_{2} s\right)\right],
\end{aligned}
$$

where the terms in the square bracket are to be evaluated at $\varepsilon=0$. The numerical evaluation of this expression at $Q=30 \mathrm{GeV}$ is plotted in fig. 1a (with $\Lambda=0.5 \mathrm{GeV}$ and $\left\langle\boldsymbol{k}_{\mathrm{T}}\right\rangle=0.5 \mathrm{GeV} / c$ ). The acollinearity distribution which follows from the modified evolution equations of ref. [11] is shown in fig. $1 \mathrm{~b}$, where the expression given by eq. (16) with a cut-off mass of $Q_{0}^{\prime}=1 \mathrm{GeV}$ is used. When compared with the result of eq. (20), no significant differences can be observed.

\section{Discussion of the result}

In order to discuss the significance of the different factors in eq. (20) we now investigate a sequence of approximations. When the coefficients $A_{2}^{\mathrm{NS}}, A_{2}^{\mathrm{gg}}, A_{2}^{\mathrm{qg}}$, and 
$A_{2}^{\mathrm{gq}}$ are put to zero, eq. (20) becomes

$$
\frac{\mathrm{d} w^{\mathrm{form}}}{\mathrm{d} \sin ^{2} \frac{1}{2} \theta}=\frac{1}{2} \int_{0}^{\infty} \mathrm{d}(b Q) b Q J_{0}\left(b Q \sin \frac{1}{2} \theta\right)\left(d\left(b, Q_{0}^{2}\right)\right)^{2} F_{\mathrm{qq}}\left(Q^{2}, Q_{0}^{2}, b\right) .
$$

We have checked that these cross sections, eqs. (20), (21), are numerically the same in the PETRA energy range. It means that the soft gluon emission from quarks dominates $\mathrm{d} w / \mathrm{d} \sin ^{2} \frac{1}{2} \theta$ at these energies.

The form factor $F_{\mathrm{qq}}$ in eq. (21) is damping the integrand in the region of large $b$; therefore, the effect of the soft gluon emission for $Q>Q_{0}$ shows up mainly at small angles of $\theta$. In fig. la we compare $\mathrm{d} w / \mathrm{d} \sin ^{2} \frac{1}{2} \theta$ at $Q=30 \mathrm{GeV}$ with the result of parton model $\left(F_{\mathrm{qq}} \equiv 1\right)$,

$$
\frac{\mathrm{d} w^{\text {parton }}}{\mathrm{d} \sin ^{2} \frac{1}{2} \theta}=\frac{1}{2} \int_{0}^{\infty} \mathrm{d}(b Q) b Q J_{0}\left(b Q \sin \frac{1}{2} \theta\right)\left(d\left(b, Q_{0}^{2}\right)\right)^{2}
$$

Indeed it can be observed that for small $\theta \lesssim 10-15^{\circ}, \mathrm{d} w / \mathrm{d} \sin ^{2} \frac{1}{2} \theta$ from eq. (20) predicts less events than the parton model.

A QED-like result can be derived from the expression eq. (21), when one takes $\alpha_{\mathrm{s}}\left(k^{2}\right)=\alpha_{\mathrm{s}}=$ const., $d\left(b, Q_{0}^{2}\right)=1$ and $Q_{0}=0$. It reads

$$
\frac{\mathrm{d} w^{\mathrm{QED}}}{\mathrm{d} \sin ^{2} \frac{1}{2} \theta}=\frac{1}{2} \int_{0}^{\infty} \mathrm{d}(b Q) b Q J_{0}\left(b Q \sin \frac{1}{2} \theta\right) \exp \left[-\frac{4 c_{\mathrm{F}}}{\pi} \alpha_{\mathrm{s}} \int_{0}^{b Q} \frac{\mathrm{d} q}{q} \ln \frac{b Q}{q}\left(1-J_{0}(q)\right)\right] \text {. }
$$

This expression has been examined in detail by Parisi and Petronzio [3] in the context of the Drell-Yan process. It is a result of the summation of real and virtual soft gluons to all orders in perturbation theory. The main features of eq. (23) are that it scales and that it does not vanish at $\theta=0$. There is a non-vanishing probability of compensating the transverse momenta of the emitted gluons such that the quarks remain undeflected.

Figs. $1 \mathrm{~b}$ and $2 \mathrm{a}$ show the prediction of $\mathrm{d} w^{\mathrm{QED}} / \mathrm{d} \sin ^{2} \frac{1}{2} \theta$ with $\alpha_{\mathrm{s}}$ evaluated at $Q=30 \mathrm{GeV}, \alpha_{\mathrm{s}}=0.184$. By comparing this QED result with $\mathrm{d} w / \mathrm{d} \sin ^{2} \frac{1}{2} \theta$ [eq. (20)] one can see from fig. $1 \mathrm{~b}$ that these two distributions are rather different for $\theta \lesssim 15^{\circ}$. It means that the effects due to the intrinsic transverse momentum $\left\langle\boldsymbol{k}_{\mathrm{T}}\right\rangle \simeq 0.50$ $\mathrm{GeV} / c$ are still present at $Q=30 \mathrm{GeV}$. Therefore, at this energy, the acollinearity distribution is not given exclusively by the form factor $F_{\mathrm{qq}}$. Instead one has to go to rather high energies $Q$, larger than $500 \mathrm{GeV}$, such that at $\operatorname{small} \theta \mathrm{d} w / \mathrm{d} \sin ^{2} \frac{1}{2} \theta$ becomes numerically insensitive to the input function $d\left(b, Q_{0}^{2}\right)$. This can be deduced by looking, e.g., at the integrand of eq. (21): at a fixed value of $(b Q) \neq 0$ the dominant contribution will be due to $F_{\mathrm{qq}}$ when the function $d\left(b, Q_{0}^{2}\right)$, which 


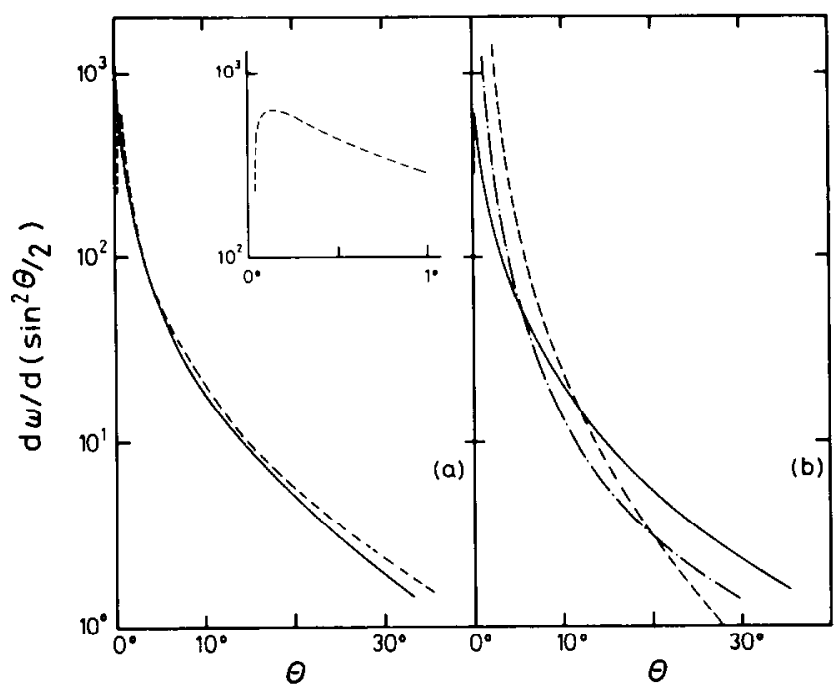

Fig. 2. The dependence of $\mathrm{d} w / \mathrm{d}\left(\sin ^{2} \frac{1}{2} \theta\right)$ on $\theta$ at $Q=30 \mathrm{GeV}$. (a) The solid curve is the QED-like result of eq. (23), the dashed one is the leading double logarithmic approximation $d w^{\text {Sudakov }} / \mathrm{d}\left(\sin ^{2} \frac{1}{2} \theta\right)$ of eq. (24). (b) The solid curve is calculated from $\mathrm{d} w^{\text {Sudakov }} / \mathrm{d}\left(\sin ^{2} \frac{1}{2} \theta\right)$ [eq. (24)], which is compared with the lowest-order calculation for the $q \bar{q}$ term of eq. (27) (dashed curve) and for the $q g$ term of eq.

(28) (dashed-dotted curve).

depends on $b\left\langle\boldsymbol{k}_{\mathrm{T}}\right\rangle$ [eq. (19)], approaches one for large $Q$. Actually this approach is very slow.

The leading double logarithmic approximation of $\mathrm{d} w$ QED $/ \mathrm{d} \sin ^{2} \frac{1}{2} \theta$ with $\alpha_{\mathrm{s}} \ln ^{2} \theta \gg 1,\left|\alpha_{\mathrm{s}} \ln \theta\right|<1$ and $\alpha_{\mathrm{s}}<1$ is extensively discussed in the recent literature $[4,6,12,15]$. In this limit all the soft gluons are confined into a cone of opening angle $\theta$. The angular distribution is expressed in terms of a Sudakov-type form factor $F_{\text {Sudakov }}$ :

$$
\frac{\mathrm{d} w^{\text {Sudakov }}}{\mathrm{d} \sin ^{2} \frac{1}{2} \theta}=\frac{\mathrm{d}}{\mathrm{d} \sin ^{2} \frac{1}{2} \theta} F_{\text {Sudakov }}\left(\sin \frac{1}{2} \theta\right)
$$

where

$$
F_{\text {Sudakov }}\left(\sin \frac{1}{2} \theta\right)=\exp \left[-\frac{c_{\mathrm{F}}}{2 \pi} \alpha_{\mathrm{s}} \ln ^{2}\left(\sin ^{2} \frac{1}{2} \theta\right)\right]
$$

In contrast to eq. (23) $\mathrm{d} w^{\text {Sudakov }} / \mathrm{d} \sin ^{2} \frac{1}{2} \theta$ vanishes for $\theta \rightarrow 0$, but its peak value $\left(\simeq \exp \left(\pi / 2 \alpha_{\mathrm{s}} c_{\mathrm{F}}\right)\right)$ at $\sin \frac{1}{2} \theta \simeq \exp \left[-\pi / 2 \alpha_{\mathrm{s}} c_{\mathrm{F}}\right]$ is very near to $\theta \simeq 0$. This is shown in fig. $2 \mathrm{a}$, where one also observes that except for $\theta<2^{\circ} \mathrm{d} w^{\mathrm{QED}} / \mathrm{d} \sin ^{2} \frac{1}{2} \theta$ and $\mathrm{d} w^{\text {Sudakov }} / \mathrm{d} \sin ^{2} \frac{1}{2} \theta$ give almost identical results.

The leading contributions considered above can be compared with the exact, but lowest order perturbative result [7]. The acollinearity distribution to order $\alpha_{\mathrm{s}}$ and 
for $\theta \neq 0$ is given by two terms where either the $q \bar{q}$ or the $q g$ pair define the acollinearity,

$$
\frac{\mathrm{d} w^{\text {pert }}}{\mathrm{d} \sin ^{2} \frac{1}{2} \theta}=\frac{\mathrm{d} w^{\mathrm{q} \overline{\mathrm{q}}}}{\mathrm{d} \sin ^{2} \frac{1}{2} \theta}+\frac{\mathrm{d} w^{\mathrm{qg}}}{\mathrm{d} \sin ^{2} \frac{1}{2} \theta}
$$

where

$$
\frac{\mathrm{d} w^{\mathrm{q} \overline{\mathrm{q}}}}{\mathrm{d} \sin ^{2} \frac{1}{2} \theta}=\frac{\alpha_{\mathrm{s}}}{2 \pi} c_{\mathrm{F}} \frac{1}{\sin ^{2} \frac{1}{2} \theta}\left[\ln \left(\sin ^{2} \frac{1}{2} \theta\right)\left(\frac{3}{\xi^{4}}-\frac{4}{\xi^{5}}\right)-\frac{4}{\xi^{4}}+\frac{1}{\xi^{3}}+\frac{1}{6 \xi^{2}}\right]
$$

and

$$
\frac{\mathrm{d} w^{\mathrm{qg}}}{\mathrm{d} \sin ^{2} \frac{1}{2} \theta}=\frac{\alpha_{\mathrm{s}}}{2 \pi} c_{\mathrm{F}}\left[\ln \left(\sin ^{2} \frac{1}{2} \theta\right) \frac{2(3-\xi)(1-2 \xi)+7}{\xi^{5}}+\frac{1}{\sin ^{2} \frac{1}{2} \theta} \frac{53 \xi^{2}-123 \xi+78}{6 \xi^{4}}\right],
$$

with $\xi=\cos ^{2} \frac{1}{2} \theta$. These two contributions are plotted in fig. $2 b$. It is evident that the leading infrared singularity, which is present in the q $\bar{q}$ term for $\theta \rightarrow 0$, disappears, when all orders of perturbation theory are summed. Furthermore fig. $2 b$ indicates that the $\mathrm{q} \overline{\mathrm{q}}$ and $\mathrm{qg}$ term become of the same size already for $\theta \simeq 20^{\circ}$. Therefore, we may estimate that only for $\theta<20^{\circ}$ is the emission of soft gluons as treated in all the above equations really the dominant mechanism-besides the intrinsic $\boldsymbol{k}_{\mathrm{T}}$ effects-- for giving rise to acollinear opposite-side hadrons.

\section{Comparison with data}

In fig. 3 the results of our approach [eq. (20)] including the $Q^{2}$ dependence are compared to the data for $(1 / \sigma) \mathrm{d} \sigma / \mathrm{d} \theta$ of the PLUTO collaboration [1]. We plot $\mathrm{d} w / \mathrm{d} \theta$ at two different energies $Q=9.4$ and $30 \mathrm{GeV}$. Because of the small-angle approximations throughout the derivations we cannot predict the size correctly; therefore, considering the region $\theta \leqslant 30^{\circ}$, we normalize the theoretical distributions to the experimental ones in this interval. At $Q=Q_{0}=9.4 \mathrm{GeV}, \mathrm{d} w / \mathrm{d} \theta$ [eq. (20)] coincides with the input given by the parton model $\mathrm{d}^{\text {parton }} / \mathrm{d} \theta$ [eq. (22)], which correctly describes the shape of the measured $(1 / \sigma) \mathrm{d} \sigma / \mathrm{d} \theta$ with $\left\langle\boldsymbol{k}_{\mathrm{T}}\right\rangle=500$ $\mathrm{MeV} / c$. At the highest available energy $Q \simeq 30 \mathrm{GeV}, \mathrm{d} w / \mathrm{d} \theta$ and $\mathrm{d} w^{\text {parton }} / \mathrm{d} \theta$ behave differently in the interesting region of $\theta<30^{\circ}$. Fig. $3 \mathrm{~b}$ shows that $\mathrm{d} w / \mathrm{d} \theta$ gives a better description of the data, which are not so strongly peaked as $\mathrm{d} w^{\text {parton }} / \mathrm{d} \theta$. Indeed the agreement is satisfactory [16]. One may therefore conclude that the data give evidence for the QCD expectation that opposite-side hadrons become more acollinear at small angles $\theta$ with increasing energy due to the multiple emission of soft gluons. 


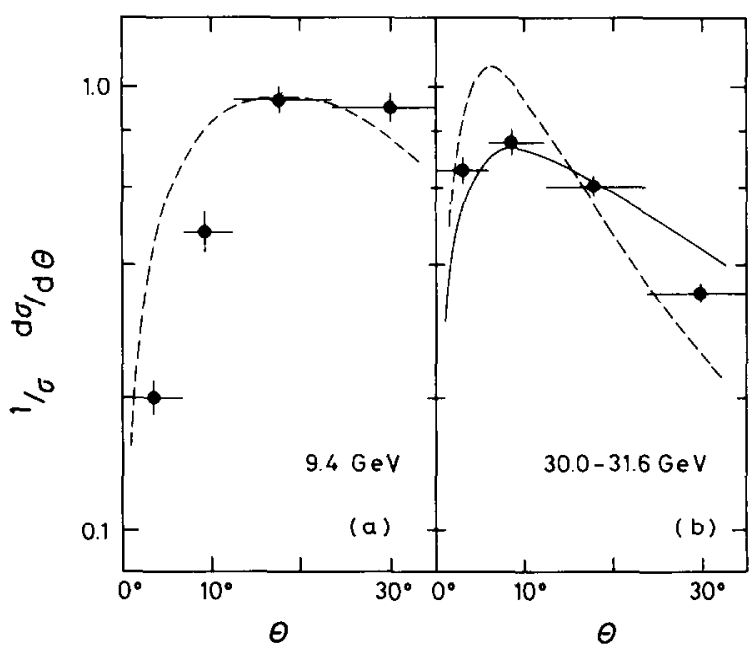

Fig. 3. The dependence of $(1 / \sigma) \mathrm{d} \sigma / \mathrm{d} \theta$ on $\theta$ (a) at $9.4 \mathrm{GeV}$ and (b) at $30 \mathrm{GeV}$. The data points are taken from ref. [1]. The dashed curves show the result for the parton model [eq. (22)], the solid curve is the result of our QCD calculation [eq. (20)].

We are grateful to J. Engels, M. Kuroda and B. Petersson for helpful discussions. We thank H. Meyer for information on the data of the PLUTO collaboration.

\section{References}

[1] Ch. Berger et al., PLUTO Collaboration, Phys. Lett. 90B (1980) 312; H. Meyer, updated data of the PLUTO collaboration, private communication

[2] A. Bassetto, M. Ciafaloni and G. Marchesini, Phys. Lett. 86B (1979) 366; Nucl. Phys. B163 (1980) 477

[3] G. Parisi and R. Petronzio, Nucl. Phys. B154 (1979) 427

[4] Yu.L. Dokshitser, D.I. Dyakonov and S.I. Troyan, Phys. Reports 58 (1980) 269; Materials of 13th Winter School of LNPI, vol. 1, Leningrad 1978, 3, SLAC-TRANS-183 (1978);

Yu.L. Dokshitser and D.I. Dyakonov, Materials of 14th Winter School of LNPI, vol. 1, Leningrad 1979, 27, DESY-L Trans. 234 (1979)

[5] D. Amati and G. Veneziano, Phys. Lett. 83B (1979) 87

[6] Yu.L. Dokshitser, D.I. Dyakonov and S.I. Troyan, Phys. Lett. 78B (1978) 290

[7] C.L. Basham, L.S. Brown, S.D. Ellis and S.T. Love, Phys. Rev. D19 (1979) 2018

[8] G. Altarelli and G. Parisi, Nucl. Phys. B126 (1977) 298

[9] R.D. Field, Perturbative quantum chromodynamics and applications to large momentum transfer processes, Caltech preprint CALT-68-739 (1979)

[10] J.F. Owens, Phys. Lett. 76B (1978) 85;

T. Uematsu, Phys. Lett. 79B (1978) 97

[11] D. Amati, A. Bassetto, M. Ciafaloni, G. Marchesini and G. Veneziano, Nucl. Phys. B173 (1980) 429

[12] G.C. Fox and S. Wolfram, A gallimaufry of $\mathrm{e}^{+} \mathrm{e}^{-}$annihilation event shapes, Caltech preprint CALT-68-723 (1979)

[13] G. Wolf, Proc. EPS Int. Conf. on High-energy physics, CERN, Geneva 1979 (CERN) 220

[14] P. Söding, Proc. EPS Int. Conf. on High-energy physics, CERN, Geneva 1979 (CERN) 271 
[15] C.L. Basham, L.S. Brown, S.D. Ellis and S.T. Love, Phys. Lett. 85B (1979) 279;

C.Y. Lo and J.D. Sullivan, Phys. Lett. 86B (1979) 327;

A. Nicolaidis, Proc. 14th Rencontre de Moriond, Les Arcs, 1979, ed. J. Tran Thanh Van, 221;

S. Wolfram, Jet development in leading log QCD, Caltech preprint CALT-68-740 (1979);

H.D. Politzer, Still QCD-ing, Caltech preprint CALT-68-745 (1979);

F. Halzen and D.M. Scott, Phys. Lett. 94B (1980) 405;

W. Marquardt and F. Steiner, Phys. Lett. 93B (1980) 480;

S.D. Ellis and W.J. Stirling, Quark form factors and leading double logarithms in QCD, Washington preprint RLO-1388-821 (1980)

[16] K. Kajantie, E. Pietarinen, Phys. Lett. 93B (1980) 269 